\title{
A linear time algorithm for the minimum Weighted Feedback Vertex Set on diamonds
}

\author{
F. Carrabs ${ }^{\text {a }}$, R. Cerulli ${ }^{\mathrm{a}, *}$ M. Gentili ${ }^{\mathrm{a}}$, G. Parlato ${ }^{\mathrm{b}}$, \\ a Dipartimento di Matematica ed Informatica, Università di Salerno, 84084 Fisciano (SA), Italy \\ b Dipartimento di Informatica ed Applicazioni, Università di Salerno, 84081 Baronissi (SA), Italy \\ Received 29 June 2004; received in revised form 26 November 2004 \\ Available online 16 January 2005 \\ Communicated by F. Meyer auf der Heide
}

\begin{abstract}
Given an undirected and vertex weighted graph $G$, the Weighted Feedback Vertex Problem (WFVP) consists in finding a subset $F \subseteq V$ of vertices of minimum weight such that each cycle in $G$ contains at least one vertex in $F$. The WFVP on general graphs is known to be NP-hard. In this paper we introduce a new class of graphs, namely the diamond graphs, and give a linear time algorithm to solve WFVP on it.
\end{abstract}

(C) 2004 Elsevier B.V. All rights reserved.

Keywords: Graph algorithms; Weighted Feedback Vertex Set; Dynamic programming; Diamond

\section{Introduction}

Given an undirected graph $G=(V, E)$, a Feedback Vertex Set (FVS) of $G$ is a subset $F \subseteq V$ of vertices such that each cycle in $G$ contains at least one vertex in $F$, i.e., the subgraph $G^{\prime}$ induced by the set $V \backslash F$ of vertices is acyclic. The Feedback Vertex Problem (FVP) consists in finding an FVS of minimum

\footnotetext{
* Corresponding author. Tel.: +39089 963326, fax: +39089 963303.

E-mail addresses: fcarrabs@unisa.it (F. Carrabs), raffaele@unisa.it (R. Cerulli),mgentili@unisa.it (M. Gentili), gparlato@unisa.it (G. Parlato).
}

cardinality. When with each vertex $v$ of $G$ is associated a weight $w(v)$ we have a vertex weighted graph. The Weighted Feedback Vertex Problem (WFVP) on a weighted graph $G$ consists in finding an FVS of minimum weight, where the weight of the set is the sum of the weights of its elements. Both FVP and WFVP have application in several areas of computer science such as circuit testing, deadlock resolution, placement of converters in optical networks, combinatorial cut design. The FVP on general graph is known to be NPhard [5]. For the WFVP the best known approximation algorithm has approximation ratio 2 (see, for example, $[2,1])$. This problem becomes polynomial when addressed on interval graphs [7], co-comparability 
graphs [3], permutation graphs [6], convex bipartite graphs [3].

In this paper we introduce a new class of graphs, namely the diamond graphs, and give a linear time algorithm to solve WFVP on it.

The sequel of the paper is organized as follows. Section 2 describes the class of diamond graphs. Section 3 contains the description of our linear time algorithm based on dynamic programming to optimally solve WFVP on diamonds. In Section 4 we will discuss how this polynomial result can be used to improve an approximated solution on a general graph, that is the object of our further research.

\section{The class of diamond graphs}

In this section we describe formally the class of diamond graphs. First we introduce the needed notation (for any additional definition and notation we refer to [4]).

Let $G=(V, E, w)$ be an undirected and vertex weighted graph, where $V$ is the set of vertices, $E$ is the set of edges, and, $w(v)$ is a positive weight associated with each vertex $v \in V$. Given a subset $X \subseteq V$ of vertices, we define its weight $W(X)$ as the sum of the weights of its elements, i.e., $W(X)=\sum_{v \in X} w(v)$. If $X=\emptyset$ then $W(X)=0$. We denote by $G \backslash X$ the subgraph of $G$ induced by the set of vertices $V \backslash X$. A tree is an acyclic and connected graph. Given a rooted tree $T$, we denote by $C_{u}$ the set of children of vertex $u$ in $T$. We define the height $h(u)$ of a vertex $u$ in $T$, recursively as follows. If $u$ is a leaf then $h(u)=0$, otherwise $h(u)=\max _{x \in C_{u}}\{h(x)\}+1$. We define the height $h(T)$ of the tree to be equal to the height of its root. Given a vertex $u$ of $T$, the subtree $T_{u}$ rooted in $u$ is the subgraph of $T$ induced by the set of vertices constituted by $u$ and its descendants in $T$.

Now we introduce the class of Diamond graphs.

Definition 1. A weighted diamond $D_{r, z}=(V, E, w)$ with apices $r$ and $z(r, z \in V)$, is an undirected and vertex weighted graph where (i) each $v \in V$ is included in at least one simple path between $r$ and $z$ and (ii) $D_{r, z} \backslash\{z\}$ is a tree.

We refer to the two vertices $r$ and $z$ of a diamond $D_{r, z}$ as, respectively, the upper and lower apex of $D_{r, z}$, and, to the subgraph $D_{r, z} \backslash\{z\}$ as the tree $T_{r}$ rooted in $r$ associated with $D_{r, z}$. Consider Fig. 1(a) as an example. We have the diamond $D_{1,10}$ with upper apex $r=1$ and lower apex $z=10$. Note that by deleting vertex $z$ we obtain the tree $T_{1}=D_{1,10} \backslash\{z\}$.

We denote by $D_{u, z}=\left(V_{u}, E_{u}, w\right)$ the subdiamond of $D_{r, z}$, with apices $u$ and $z$, induced by the vertices of $T_{u}$ and vertex $z$. We define the height $h\left(D_{u, z}\right)$ of the diamond $D_{u, z}$ to be equal to the height of the associated tree $T_{u}$.

Finally, observe that given a diamond $D_{r, z}$, we can see it as composed by the upper apex $r$ and the subdiamonds $D_{r_{i}, z}$, for each $r_{i} \in C_{r}$, where all of them have the common lower apex $z$. Consider again

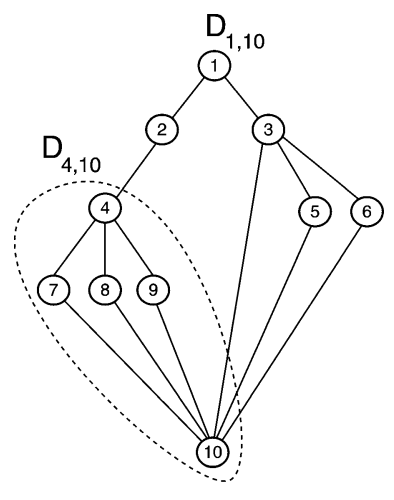

(a)

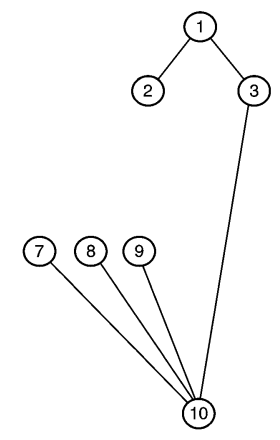

(b)

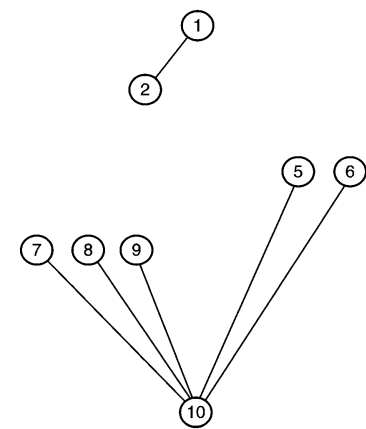

(c)

Fig. 1. (a) A diamond with apices $r=1$ and $z=10$. (b) The acyclic subgraph $D_{1,10} \backslash \hat{F}$ with a single path between vertices 1 and 10 when $\hat{F}=\{4,5,6\}$ and (c) the subgraph $D_{1,10} \backslash \hat{F}$, with $\hat{F}=\{3,4\}$ without any path between vertices 1 and 10 . 
Fig. 1(a). The subgraph composed by the vertex set $\{4,7,8,9,10\}$ is a diamond with apices $r=4$ and $z=10\left(D_{4,10}\right)$, whose associated tree is the subtree $T_{4}$ of $T_{1}$. The height of $T_{4}$ is $h\left(T_{4}\right)=h(4)=1$ that is also the height of $D_{4,10}$. Moreover, we can see $D_{1,10}$ as composed by vertex 1 and the two subdiamonds $D_{2,10}$ and $D_{3,10}$.

\section{The dynamic programming algorithm}

To simplify notation, in the rest of the section we denote a diamond $D_{r, z}$ just as $D_{r}$, since all considered diamonds have the same lower apex $z$.

In this section, we propose a linear time algorithm to solve the WFVP on a diamond based on dynamic programming. We will introduce two new problems on diamonds (the Path problem and the NoPath problem) and show how to obtain a minimum weighted FVS on $D_{r}$ by the optimum solutions of these two problems. We then prove they have an optimal substructure property that allows us to optimally solve them by means of dynamic programming.

In the sequel of the paper we will consider a diamond $D_{r}$ with upper apex $r$ and lower apex $z$. To better clarify the role of the two new problems in our resolution algorithm, we state now the following observation.

Observation 1. By definition of diamond, the set $F=$ $\{z\}$ is an FVS of $D_{r}$. Hence, an optimum solution $F^{*}$ of WFVP on $D_{r}$ is such that, either it is composed of the single vertex $z$ (i.e., $F^{*}=\{z\}$ ) or, it does not contain vertex $z$ and it is such that $W\left(F^{*}\right) \leqslant w(z)$. Therefore, the WFVP on a diamond can be solved, first by looking for the minimum FVS, say $\hat{F}$, that does not contain vertex $z$, and then, by choosing the minimum weight set between $\hat{F}$ and $\{z\}$.

Let $\hat{F} \subseteq V \backslash\{z\}$ be an FVS on $D_{r}$. Note that the subgraph $D_{r} \backslash \hat{F}$ either contains a single simple path between $r$ and $z$ or it does not contain any of such a path, as it is stated by the following proposition.

Proposition 1. Given a diamond $D_{r}$ with apices $r$ and $z$, if $\hat{F} \subseteq V \backslash\{z\}$ is an $F V S$ of $D_{r}$, then there exists at most one path between $r$ and $z$ in $D_{r} \backslash \hat{F}$.
Proof. By contradiction let us suppose that there exist two distinct paths $p_{1}$ and $p_{2}$ between $r$ and $z$ in $D_{r} \backslash \hat{F}$. Let $v \neq z$ be the last vertex of the common longest subpath of $p_{1}$ and $p_{2}$ starting from $r$. Then the subpaths from $v$ to $z$ of $p_{1}$ and $p_{2}$, joined together, form a cycle in $D_{r} \backslash \hat{F}$, but this is a contradiction since $D_{r} \backslash \hat{F}$ must be acyclic being $\hat{F}$ an FVS of $D_{r}$.

For example, consider again the diamond in Fig. 1. The set $\hat{F}=\{4,5,6\}$ is an FVS that does not contain vertex $z$ and such that there exists the simple path $P=$ $\{1,3,10\}$ connecting $r$ and $z$ in $D_{r} \backslash \hat{F}$ (see Fig. 1(b)). The set $\hat{F}=\{3,4\}$ is an FVS that does not contain $z$ and such that there is no path connecting $r$ and $z$ in $D_{r} \backslash \hat{F}$ (see Fig. 1(c)).

Now, we are ready to define two new problems that will be useful to solve WFVP on $D_{r}$.

Path problem. Given a diamond $D_{r}$, find a subset $F_{r}^{+} \subseteq V \backslash\{z\}$ of minimum weight such that (i) $D_{r} \backslash F_{r}^{+}$ does not contain cycles, and (ii) there exists exactly a single path in $D_{r} \backslash F_{r}^{+}$between $r$ and $z$.

NoPath problem. Given a diamond $D_{r}$, find a subset $F_{r}^{-} \subseteq V \backslash\{z\}$ of minimum weight such that (i) $D_{r} \backslash F_{r}^{-}$ does not contain cycles, and (ii) there does not exist a path in $D_{r} \backslash F_{r}^{-}$between $r$ and $z$.

From the above observations it follows that the optimum solution $F^{*}$ of WFVP on $D_{r}$ is such that:

$$
W\left(F^{*}\right)=\min \left\{w(z), W\left(F_{r}^{+}\right), W\left(F_{r}^{-}\right)\right\}
$$

and, therefore we have either $F^{*}=\{z\}$ or $F^{*}=F_{r}^{+}$or $F^{*}=F_{r}^{-}$.

Hence, we are interested now in solving both Path and NoPath problems.

\subsection{Optimal substructure and recursion rules}

In this section, we conjunctly characterize the structure of an optimal solution for both Path and NoPath problems. We recall that a problem has the optimal substructure property if any optimal solution to the problem contains within it optimal solutions to subproblems [4]. We will see that both Path and NoPath problems, defined on $D_{r}$, have an optimal substructure property by considering as subproblems those of determining the solution to Path and NoPath 
on the subdiamonds $D_{u}$, for each $u \in V \backslash\{z\}$. Let us denote by $F_{u}^{+}$and $F_{u}^{-}$the optimal solutions of Path and NoPath problem, respectively, on $D_{u}$. Now, we prove that the optimal solution $F_{u}^{+}\left(F_{u}^{-}\right)$contains, for each $u_{i} \in C_{u}$, either $F_{u_{i}}^{+}$or $F_{u_{i}}^{-}$.

Proposition 2. Given the optimum solution $F_{u}^{+}$on $D_{u}$, then each set $F_{u_{i}}=F_{u}^{+} \cap V_{u_{i}}, u_{i} \in C_{u}$, is an optimum solution to either Path problem or NoPath problem on $D_{u_{i}}$.

Proof. By feasibility of $F_{u}^{+}$, it follows that $z \notin F_{u_{i}}$ and $D_{u_{i}} \backslash F_{u_{i}}$ is acyclic. Then, the set $F_{u_{i}}$ respects condition (i) for both Path problem and NoPath problem on the subdiamond $D_{u_{i}}$. Now, two cases, based on the fact that either there exists a path between $u_{i}$ and $z$ on $D_{u_{i}} \backslash F_{u_{i}}$ or not, may occur. If there is such a path, then $F_{u_{i}}$ is the optimum solution set for Path problem on $D_{u_{i}}$, i.e., $F_{u_{i}}=F_{u_{i}}^{+}$. Indeed, if there were another feasible set $F_{u_{i}}^{\prime}$ for Path problem on $D_{u_{i}}$ such that $W\left(F_{u_{i}}^{\prime}\right)<W\left(F_{u_{i}}\right)$, we could obtain the set $F_{u}^{\prime+}=\left(F_{u}^{+} \backslash F_{u_{i}}\right) \cup F_{u_{i}}^{\prime}$ that is feasible for Path problem on $D_{u}$ and such that $W\left(F_{u}^{\prime+}\right)<W\left(F_{u}^{+}\right)$obtaining a contradiction. If there does not exit any path between $u_{i}$ and $z$ on $D_{u_{i}} \backslash F_{u_{i}}$, then by applying a similar reasoning it follows that $F_{u_{i}}=F_{u_{i}}^{-}$.

The same considerations can be made for the optimum solution $F_{u}^{-}$of the NoPath problem as stated by the following proposition.

Proposition 3. Given the optimum solution $F_{u}^{-}$on $D_{u}$, then each set $F_{u_{i}}=F_{u}^{-} \cap V_{u_{i}}, u_{i} \in C_{u}$, is an optimum solution to either Path problem or NoPath problem on $D_{u_{i}}$.

Following Lemmas 1 and 2, we describe how to build the weights of sets $F_{u}^{+}$and $F_{u}^{-}$on $D_{u}$, by considering the value of optimum solution of Path and NoPath problems on $D_{x}$, for each $x \in C_{u}$. In the next section we will use these values to construct the optimum sets $F_{r}^{+}$and $F_{r}^{-}$.

\section{Lemma 1.}

Case A: $h(u)=0$. $W\left(F_{u}^{+}\right)=0$.

Case B: $h(u)>0$ and $(u, z) \in E$. $W\left(F_{u}^{+}\right)=\sum_{x \in C_{u}} W\left(F_{x}^{-}\right)$.
Case C: $h(u)>0$ and $(u, z) \notin E$.

$$
W\left(F_{u}^{+}\right)=\min _{x \in C_{u}}\left\{W\left(F_{x}^{+}\right)+\sum_{y \in C_{u} \backslash\{x\}} W\left(F_{y}^{-}\right)\right\} .
$$

Proof. Case A is easily verified since $F_{u}^{+}=\emptyset$.

Case B: if $(u, z) \in E$, since $u \notin F_{u}^{+}$, then the path connecting $u$ and $z$ in $D_{u} \backslash F_{u}^{+}$is composed by the single edge $(u, z)$. Thus, to avoid cycles in $D_{u} \backslash F_{u}^{+}$, the set $F_{u}^{+}$is obtained, by Propositions 1 and 2, by the union of the optimum sets $F_{x}^{-}, x \in C_{u}$, and we obtain $W\left(F_{u}^{+}\right)=\sum_{x \in C_{u}} W\left(F_{x}^{-}\right)$.

Case C: if $(u, z) \notin E$, since $u \notin F_{u}^{+}$, in order to have a path between $u$ and $z$ in $D_{u} \backslash F_{u}^{+}$and by applying Propositions 1 and 2, the optimum set is obtained by the minimum weight union of exactly one set $F_{x}^{+}$, $x \in C_{u}$, and, $F_{y}^{-}, y \in C_{u} \backslash\{x\}$. Therefore, $W\left(F_{u}^{+}\right)=$ $\min _{x \in C_{u}}\left\{W\left(F_{u}^{+}\right)+\sum_{y \in C_{u} \backslash\{x\}} W\left(F_{y}^{-}\right)\right\}$.

\section{Lemma 2.}

Case A: $h(u)=0$.

$$
W\left(F_{u}^{-}\right)=w(u) .
$$

Case B: $h(u)>0$ and $(u, z) \in E$.

$$
W\left(F_{u}^{-}\right)=w(u)+\sum_{x \in C_{u}} \min \left\{W\left(F_{x}^{-}\right), W\left(F_{u}^{+}\right)\right\} .
$$

Case C: $h(u)>0$ and $(u, z) \notin E$.

$$
\begin{gathered}
W\left(F_{u}^{-}\right)=\min \left\{w(u)+\sum_{x \in C_{u}} \min \left\{W\left(F_{x}^{-}\right), W\left(F_{x}^{+}\right)\right\},\right. \\
\left.\sum_{x \in C_{u}} W\left(F_{x}^{-}\right)\right\} .
\end{gathered}
$$

Proof. Case A is easily verified since $F_{u}^{-}=\{u\}$.

Case B: if $(u, z) \in E$, then $u \in F_{u}^{-}$otherwise there would be a path between $u$ and $z$ in $D_{u} \backslash F_{u}^{-}$. Therefore, by applying Propositions 1 and 3, we select on each subdiamond $D_{x}, x \in C_{u}$, the minimum weighted set among $F_{x}^{+}$and $F_{x}^{-}$. Then $W\left(F_{u}^{-}\right)=$ $w(u)+\sum_{x \in C_{u}} \min \left\{W\left(F_{x}^{-}\right), W\left(F_{x}^{+}\right)\right\}$.

Case C: if $(u, z) \notin E$, then $u$ can either belong to $F_{u}^{-}$or not. If $u \in F_{u}^{-}$, there is no path between $u$ and $z$ in $D_{u} \backslash F_{u}^{-}$, thus we have the same solution as for Case B. If $u \notin F_{u}^{-}$then to avoid paths between the apices in $D_{u} \backslash F_{u}^{-}$, the optimum set is obtained by the union of the optimum solutions of the NoPath problem on the subdiamonds $D_{x}$ for each child $x$ of vertex $u$, therefore we would have $W\left(F_{u}^{-}\right)=\sum_{x \in C_{u}} W\left(F_{u}^{-}\right)$. Thus, for this case, $W\left(F_{u}^{-}\right)=\min \left\{w(u)+\sum_{x \in C_{u}} \min \left\{W\left(F_{x}^{-}\right), W\left(F_{x}^{+}\right)\right\}\right.$, $\left.\sum_{x \in C_{u}} W\left(F_{x}^{-}\right)\right\}$. 
According to lemmas above, the computation of the optimum weighted FVS value can be carried out by a dynamic programming algorithm. The algorithm scans all the vertices of $T_{r}$ through a postorder visit starting from the root node $r$. At each vertex $u$, both weight $W\left(F_{u}^{+}\right)$and $W\left(F_{u}^{-}\right)$, to Path and NoPath problems on $D_{u}$ are computed. Once that the weights of these sets are computed for the upper apex $r$, the optimum set among $\{z\}, F_{r}^{+}$and $F_{r}^{-}$is chosen.

The computational complexity of the algorithm, sketched above, is given by the sum of the computational complexity needed to compute $W\left(F_{u}^{+}\right)$and $W\left(F_{u}^{-}\right)$, for each vertex $u$ of the tree $T_{r}$. The computation involved in both cases A of Lemmas 1 and 2 takes $\mathrm{O}(1)$ time. For cases $\mathrm{B}$ it takes $\mathrm{O}\left(\left|C_{u}\right|\right)$ time. Indeed, the computation of $W\left(F_{u}^{+}\right)$is carried out by computing the sum of $\left|C_{u}\right|$ values, while, to compute $W\left(F_{u}^{-}\right),\left|C_{u}\right|$ minimum operations between two values are carried out and the sum of $\left|C_{u}\right|+1$ values is computed. It follows directly that the evaluation of $W\left(F_{u}^{-}\right)$, for case $\mathrm{C}$ of Lemma 2 , takes $\mathrm{O}\left(\left|C_{u}\right|\right)$ time. Finally, observe that, to compute $W\left(F_{u}^{+}\right)$for case C of Lemma 1, we can evaluate $\min _{x \in C_{u}}\left\{S-W\left(F_{x}^{-}\right)+\right.$ $\left.W\left(F_{x}^{+}\right)\right\}$, where $S=\sum_{x \in C_{u}} W\left(F_{x}^{-}\right)$, which can be accomplished in $\mathrm{O}\left(\left|C_{u}\right|\right)$ time. Thus, the computational complexity of the algorithm is given by

$$
\sum_{u \in V \backslash\{z\}} \mathrm{O}\left(\left|C_{u}\right|\right)=\mathrm{O}(|E|)=\mathrm{O}(|V|),
$$

where the last relation follows from the fact that for any diamond $|E| \leqslant 2|V|$. The above observations prove the following theorem.

Theorem 1. The solution value of the weighted feedback vertex set problem on diamonds can be computed $\mathrm{O}(|V|)$ time.

The detailed dynamic programming algorithm is given in Fig. 2.

\subsection{Construction of $F_{r}^{+}$and $F_{r}^{-}$sets}

In this section we describe the linear time recursive procedure, Build_Solution, to build the feedback vertex set whose optimum value is found by our dynamic

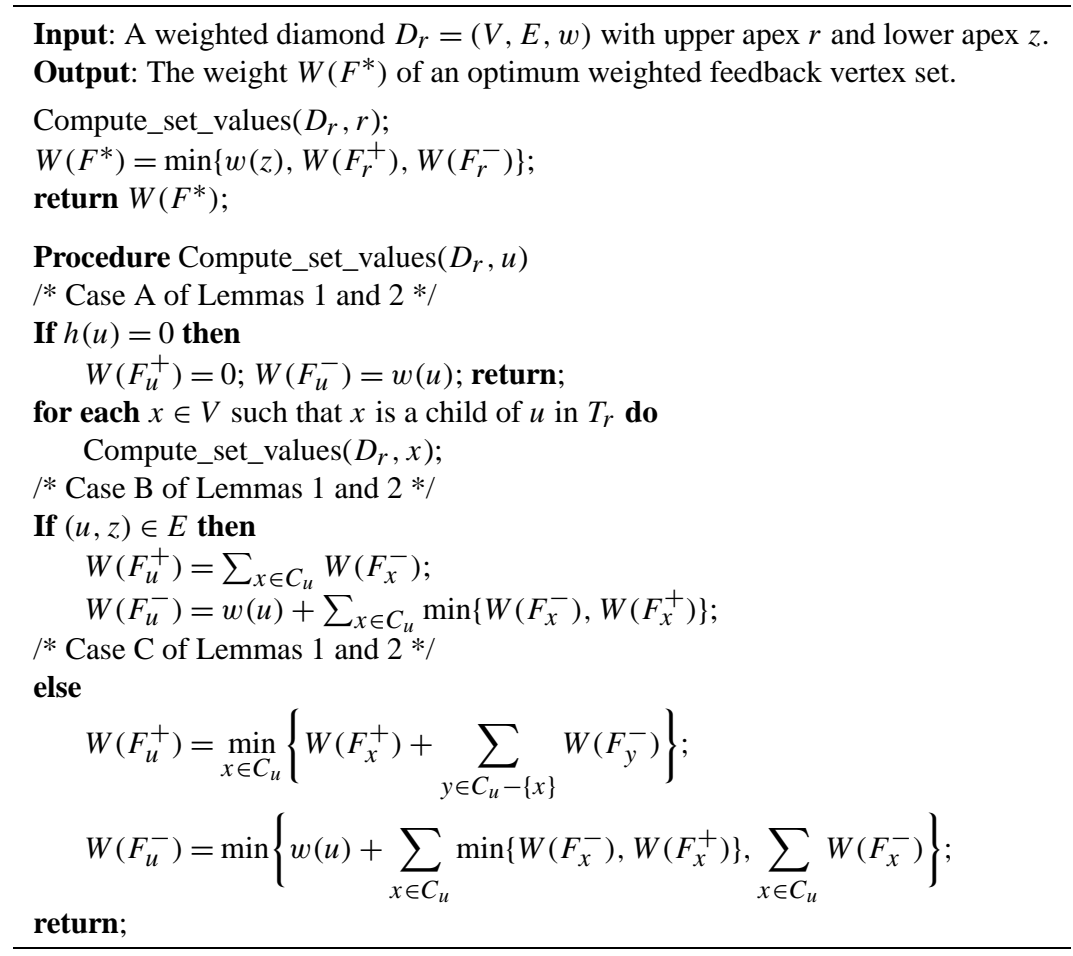

Fig. 2. The dynamic programming algorithm. 


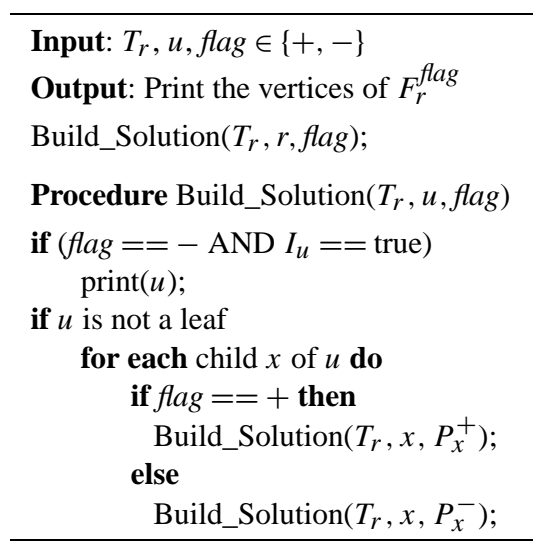

Fig. 3. Build_Solution procedure.

programming algorithm presented in the previous section. By Lemmas 1 and 2, given a vertex $u$ of $T_{r}$, an easy way to build the optimal sets $F_{u}^{-}$and $F_{u}^{+}$is to store the optimal sets $F_{u_{i}}^{-}$and $F_{u_{i}}^{+}$for each $u_{i} \in C_{u}$. However, from an implementation point of view, this choice would require both the storage of a large quantity of data and the use of complex data structures to manage sets. Now, we describe a more efficient strategy to build these optimum sets. In order to do that, we associate with each vertex $u$ of $T_{r}$ a boolean variable $I_{u}$ that holds TRUE if and only if $u \in F_{u}^{-}$. Moreover, for each vertex $u \neq r$ of $T_{r}$, we associate two variables $P_{u}^{+}$and $P_{u}^{-}$with values from $\{+,-\}$. In more detail, given $x \in C_{u}, P_{x}^{+}=+\left(P_{x}^{-}=+\right)$if $F_{x}^{+} \subseteq F_{u}^{+}$ $\left(F_{x}^{+} \subseteq F_{u}^{-}\right)$and $P_{x}^{+}=-\left(P_{x}^{-}=-\right)$if $F_{x}^{-} \subseteq F_{u}^{+}$ $\left(F_{x}^{-} \subseteq F_{u}^{-}\right)$.

These variables are set during the execution of the dynamic programming algorithm and are used by the procedure Build_Solution shown in Fig. 3. This procedure takes as input the tree $T_{r}$, a vertex $u \in T_{r}$ and a flag $\in\{+,-\}$, and returns either the optimum set $F_{u}^{+}$ or $F_{u}^{-}$according to the value of flag.

For example, by calling Build_Solution $\left(T_{r}, u,+\right)$ the set $F_{u}^{+}$is returned. Note that this set (by Propositions 2 and 3) contains, for each child $x$ of $u$, either $F_{x}^{+}$or $F_{x}^{-}$, and this information is described by the value of $P_{x}^{+}$. Therefore, the procedure is recursively called on each child $x$ of $u$ with parameters $T_{r}, x, P_{x}^{+}$. A similar reasoning is applied when Build_Solution is called to build $F_{u}^{-}$. However, since in this case $u$ can either belong to $F_{u}^{-}$or not, the value of the variable $I_{u}$ has to be considered too.

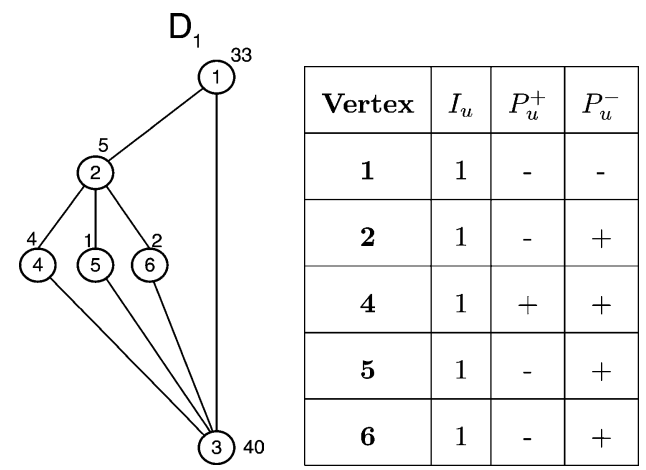

Fig. 4. On the left a diamond whose optimum weighted FVS is $F^{*}=\{2\}$ is given, and on the right, the values of the labels associated with each vertex are shown.

Consider as an example the diamond in Fig. 4. The upper apex is $r=1$ and the lower apex is $z=3$, the label on each vertex denotes its weight, for example, vertex 1 has weight $w(1)=33$. The optimum weighted FVS is $F^{*}=\{2\}$ whose optimum weight is $W\left(F^{*}\right)=\min \left\{w(z), W\left(F_{1}^{+}\right), W\left(F_{1}^{-}\right)\right\}$, where $F_{1}^{+}=$ $\{2\}$ and $F_{1}^{-}=\{1,5,6\}$. The values of the labels to build the optimum sets $F_{u}^{+}$and $F_{u}^{-}$are given in Fig. 4 .

The optimum sets $F_{u}^{-}$associated with the vertices $4,5,6$, are, respectively, $F_{4}^{-}=\{4\}, F_{5}^{-}=\{5\}, F_{6}^{-}=$ $\{6\}$, and therefore, $I_{4}=I_{5}=I_{6}=$ true. The set $F_{2}^{+}=$ $\{5,6\}$ is computed by selecting $F_{4}^{+}=\emptyset, F_{5}^{-}=\{5\}$ and $F_{6}^{-}=\{6\}$, therefore, $P_{4}^{+}=+, P_{5}^{+}=-$and $P_{6}^{+}=-$.

It is easy to see that the time complexity of procedure Build_Solution is linear. Hence, we have the main result of this section.

Theorem 2. The weighted feedback vertex set problem on diamonds can be solved in $\mathrm{O}(|V|)$ time.

\section{Conclusion and further research}

In this paper we have presented the family of diamond graphs where it is possible to solve WFVP in linear time. We described a dynamic programming algorithm to compute both the value and the vertices that compose the optimal solution in $\mathrm{O}(n)$ time. Object of our further research is both (i) the study of the larger class of multidiamond graphs (diamonds with multi-upper and/or lower apices), and (ii) the use of our exact algorithm on diamonds to improve the approximated solution returned by existing heuristics that solve WFVP on general graphs. To better clarify 
this idea, let $G$ be a graph and $F$ be an approximate FVS returned by a given approximation algorithm. We could improve the solution of the given set $F$ by substituting one or more of its vertices, say $F^{\prime} \subseteq F$, with a set $S \subseteq V \backslash F$ of less weight such that the resulting new set is an FVS. Consider, for example, the acyclic subgraph $G \backslash F=T$, and, assume to add to it the vertex $z \in F$. The resulting graph is, after appropriate reduction operations, either a diamond or a multidiamond and by applying our algorithm we could improve the initial FVS.

\section{References}

[1] V. Bafna, P. Berman, T. Fujito, Constant ratio approximations of the weighted feedback vertex set problem for undirected graphs, in: ISAAC95, Algorithms and Computation, in: Lecture Notes in Comput. Sci., vol. 1004, Springer-Verlag, Berlin, 1995, pp. $142-151$.

[2] A. Becker, D. Geiger, Approximation algorithms for the loop cutset problem, in: Proc. 10th Conf. on Uncertainty in Artificial Intelligence, 1994, pp. 60-68.

[3] M.S. Chang, Y.D. Liang, Minimum feedback vertex sets in cocomparability graphs and convex bipartite graphs, Acta Inform. 34 (1997) 337-346.

[4] T.H. Cormen, C.E. Leiserson, R.L. Rivest, Introduction to Algorithms, MIT Press, Cambridge, MA, 2001.

[5] M.R. Garey, D.S. Johnson, Computers and Intractability: A Guide to the Theory of NP-Completeness, Freeman, San Francisco, CA, 1979.

[6] Y.D. Liang, On the feedback vertex set problem in permutation graphs, Inform. Process. Lett. 52 (1994) 123-129.

[7] C.L. Lu, C.Y. Tang, A linear-time algorithm for the weighted feedback vertex problem on interval graphs, Inform. Process. Lett. 61 (1997) 107-111. 